\title{
Matrix reference materials development for food safety applications in Philippine products
}

\author{
Benilda Ebarvia ${ }^{1}$, Sharlene Cabanilla ${ }^{1}$, Aaron Dacuya ${ }^{1}$, Alleni Tongson ${ }^{1}$, Cyril Cortez ${ }^{1}$, Kim Christopher \\ Aganda $^{1}$, Natividad Mamplata ${ }^{1}$
}

${ }^{1}$ Industrial Technology Development Institute, Department of Science and Technology (ITDI-DOST), Taguig City, Philippines

\begin{abstract}
The Philippine Industrial Technology Development Institute (ITDI) - Department of Science and Technology pioneered the development of reference materials (RMs) as part of its vision to establish metrological traceability and to support the RM needs of chemical testing laboratories in the country. Food safety of Philippine products were prioritised in this activity as these could affect the health of consumers as well as trade issues. The focus of this study was on contaminants like histamine and benzoic acid in canned tuna and mango juice, respectively. The materials were prepared and characterised, and they were found to be homogeneous and stable for at least six months. Higher order methods like the isotope dilution technique, coupled with liquid chromatography-triple quadrupole mass spectrometry, were used to assign reference values to the RMs, and the results were cross-checked using gravimetric techniques for high-performance liquid chromatography analysis. Certified RMs purchased from metrology institutes were also used to further support traceability of measurements. These steps resulted in RMs for histamine and benzoic acid in Philippine products, and ITDI disseminated the measurement traceability to local laboratories through proficiency testing schemes organised and conducted for the analytes.
\end{abstract}

\section{Section: RESEARCH PAPER}

Keywords: Reference Material; Food Contaminant; Histamine; Benzoic Acid; Food Safety

Citation: Benilda Ebarvia, Sharlene Cabanilla, Aaron Dacuya, Alleni Tongson, Cyril Cortez, Kim Christopher Aganda, Natividad Mamplata, Matrix Reference Materials Development for Food Safety Application in Philippine Products, Acta IMEKO, vol. 9, no. 3, article 12, September 2020, identifier: IMEKO-ACTA09 (2020)-03-12

Section Editor: Claudia Zoani, ENEA, Italy

Received December 29, 2017; In final form July 15, 2020; Published September 2020

Funding: This work was supported by Department of Science and Technology (DOST) and the Philippine Council for Industry, Energy and Emerging Technology for Research and Development (PCIEERD), Philippines

Corresponding author: Benilda S. Ebarvia, e-mail: bsebarvia@yahoo.com

\section{INTRODUCTION}

The need to ensure comparable and traceable chemical measurements is important for a country engaging in global trade. This need requires the establishment of a national chemical measurement organisation tasked with improving the capability for accurate measurements throughout the country. Its sub-tasks could include the provision of certified reference materials (CRMs) and general reference materials (RMs), the conduct of proficiency testing (PT) schemes and the accreditation for ISO/IEC 17025. The organisation could guarantee the quality of chemical measurements performed within the country and increase the confidence in such measurements abroad.

As part of a vision to establish such an organisational infrastructure for chemical metrology in the Philippines, the Industrial Technology Development Institute (ITDI) Department of Science and Technology (DOST) pioneered the development of RMs to support the needs of chemical testing laboratories in the country. Food safety issues were prioritised in this activity since food chemicals could affect the health of consumers and the level of some substances in food has been a reason for detention of some Philippine export products abroad.

This study focused on the development of RMs for measuring histamine in canned tuna and benzoic acid in mango juice. These two chemicals are vital criteria for export food products as other countries have based their import approval decisions on the levels of these chemicals. Further, the monitoring of these two chemicals are essential for quality control and food safety in general. CRMs for benzoic acid have been developed for orange juice [1], soy sauce [2] and quasi-drug drinks [3]; however, currently, there are no Philippine RMs or PT schemes available for benzoic acid in mango juice, nor are there RMs or PT schemes for histamine levels in canned tuna available. CRMs for histamine in fish products have not been reported. Included in this study are homogeneity and stability studies, RM characterisation, and the process for conducting PT schemes for these substances. 


\section{EXPERIMENTAL}

\subsection{Chemicals and Materials}

The calibration standards for benzoic acid were SRM 350b Benzoic Acid, 99.9994\% (National Institute of Standards and Technology (NIST, USA) and HRM-1002A Benzoic Acid (Health Science Authority (HSA), Singapore). The CRMs for benzoic acid were HRM-1001A Benzoic Acid in orange juice (HSA) and isotopically labelled analogue benzoic acid ring-D5 (Cambridge Isotope Laboratory, CIL). Mass spectrometer (MS)grade solvents and high-performance liquid chromatography (HPLC)-grade solvents were used. For histamine, the calibration standards were histamine standard (Sigma Aldrich, Singapore) and histamine dihydrochloride (National Institute of Metrology China). Isotopically labelled analogue histamine dihydrochlorideD4 (CIL) was used as an internal standard. Brij-35, mercaptoethanol, o-phthaldialdehyde (OPA), and 1-octanesulfonic acid sodium salt - used in the HPLC analysis were all obtained from Sigma Aldrich.

\subsection{Measurement of benzoic acid and histamine by HPLC}

For benzoic acid analysis, the HPLC (Shimadzu Prominence LC-20A with UV - VIS Detector, set at $234 \mathrm{~nm}$ ) with Waters C18 Sunfire $(3.5 \mu \mathrm{m}, \varnothing 4.6 \mathrm{~mm} \times 150 \mathrm{~mm})$ column was used following a validated, in-house-developed method [4].

For histamine analysis, HPLC with Waters C18 Sunfire column and fluorescence detector $(340 \mathrm{~nm}$ excitation and 445 $\mathrm{nm}$ emission) was utilised after post-column derivatisation with OPA [5].

\subsection{Preparation of reference material}

The RM for benzoic acid in mango juice was prepared using locally made commercial mango juice drinks, all manufactured on the same date. The juice was mixed, centrifuged, filtered, spiked with benzoic acid and pasteurised. The hot juice was poured into $100-\mathrm{mL}$ amber bottles, which were then sealed, labelled, shrink wrapped and stored at $4{ }^{\circ} \mathrm{C}$.

The RM for histamine was prepared from canned tuna which was homogenised until a paste-like consistency was obtained. The tuna sample was spiked with a set amount of histamine and it was thoroughly mixed. The spiked tuna sample $(80 \mathrm{mg})$ was packed into a $100-\mathrm{ml}$ amber bottle with screw cap. The packed samples were properly labelled and sterilised at $115.6^{\circ} \mathrm{C}$ for about 3 hours and stored at $4{ }^{\circ} \mathrm{C}$ after cooling.

\subsection{Assessment of homogeneity and stability}

The assessment of homogeneity was carried out after 10 bottles from the batch were selected by stratified random sampling. Two subsamples were taken from each bottle for measurement. Statistical evaluation was based on the IUPAC (International Union of Pure and Applied Chemistry) Harmonised Protocol [6], and the stability of RMs was also considered. A short-term stability test using isochronous design

Table 1. Summary of validation results obtained by HPLC method for the matrix RMs.

\begin{tabular}{lcc}
\hline \multicolumn{1}{c}{ Parameter } & $\begin{array}{c}\text { Benzoic acid } \\
(\boldsymbol{n}=\mathbf{1 0})\end{array}$ & $\begin{array}{c}\text { Histamine } \\
(\boldsymbol{n}=\mathbf{1 0})\end{array}$ \\
\hline Repeatability, \% RSD & 1.08 & 2.1 \\
Trueness, \% recovery & 99.3 & 101.6 \\
Linearity, $r$ & 0.9999 & 0.9997 \\
LOD, mg/kg & 0.0161 & 0.0537 \\
LOQ, mg/kg & 0.0537 & 0.0503 \\
\hline
\end{tabular}

was carried out for three weeks at three temperatures: $4^{\circ} \mathrm{C}$, $30{ }^{\circ} \mathrm{C}$ and a simulated transport temperature of about $40{ }^{\circ} \mathrm{C}$. Two randomly selected bottles were transferred from the control temperature to each temperature setting. After the study period, all bottles were collected and analysed under repeatability conditions. For long term stability, three bottles were analysed at designated intervals: one, three and six months. The statistical evaluation was based on ISO Guide 35 [7].

\subsection{Value assignment of reference material}

Liquid chromatography-isotope dilution mass spectrometry (LC-IDMS) with gravimetric sample preparation was used for value assignment of the prepared RMs. The mass fraction of the measurand was calculated based on the equation below [8]:

$$
C=\frac{m_{\text {is-sp,sample }} \cdot m_{\text {std }} \cdot A R_{\text {sample }} \cdot C_{\text {stock,std }}}{m_{\text {sample }} \cdot A R_{\text {std }} \cdot m_{\text {is-sp,std }}}
$$

where $C$ is the concentration of the analyte in the sample $(\mathrm{mg} / \mathrm{kg}), m_{\text {is-sp, sample }}$ is the mass of isotopically labelled analyte solution spiked into the sample (g), $m_{\text {std }}$ is the mass of the analyte standard solution added into the calibration blend solution $(\mathrm{g})$, $A R_{\text {sample }}$ is the observed response ratio of the of the analyte/isotope for the sample solution, $C_{\text {stock, std }}$ is the concentration of the analyte standard solution $(\mathrm{mg} / \mathrm{kg}), m_{\text {sample }}$ is the mass of sample taken for analysis $(\mathrm{g}), A R_{\mathrm{std}}$ is the observed response ratio of the of the analyte/isotope in the calibration blend solution and $M_{\mathrm{is}-\mathrm{sp} \text {, std }}$ is the mass of isotopically labelled analyte solution added into the calibration blend solution (g).

Liquid chromatography-triple quadrupole mass spectrometry (Agilent $1290 \mathrm{LC}$ system) coupled to a $6460 \mathrm{LC} / \mathrm{MS}$ Triple Quadrupole (Agilent Technologies, Inc.) with Agilent Zorbax Eclipse Plus C18 column $(3.5 \mu \mathrm{m}, \varnothing 4.6 \mathrm{~mm} \times 100 \mathrm{~mm})$ were employed for the benzoic acid. The sample was diluted with a mobile phase solution after the addition of the benzoic acid ringD5 solution. For histamine, the separation was achieved by using the Agilent Poroshell 120 HILIC column $(2.7 \mu \mathrm{m}, \varnothing 2.1 \mathrm{~mm} \times$ $100 \mathrm{~mm}$ ). Sample extraction was based on Agilent Application Note [9]. Histamine dihydrochloride-D4 was used as the internal standard.

\section{RESULTS AND DISCUSSION}

\subsection{Method Validation}

The HPLC and LC-IDMS methods for benzoic acid and histamine with gravimetric sample preparation were validated, and the results are tabulated in Table 1 and Table 2, respectively. The use of CRM HRM-1001A Benzoic Acid in Orange Juice for benzoic acid analysis established the metrological traceability to the International System of Units (SI). The results are within the criteria set by AOAC for repeatability, trueness (\% recovery) and linearity for the concentration range of $5-100 \mathrm{mg} / \mathrm{kg}$ [10]. The LOD and LOQ obtained were lower than the ranges of sample.

Table 2. Summary of validation results obtained by LC-IDMS.

\begin{tabular}{lcc}
\hline \multicolumn{1}{c}{ Parameter } & $\begin{array}{c}\text { Benzoic acid } \\
(\boldsymbol{n}=\mathbf{1 0})\end{array}$ & $\begin{array}{c}\text { Histamine } \\
(\boldsymbol{n}=\mathbf{1 0})\end{array}$ \\
\hline Repeatability, \% RSD & 1.21 & 1.35 \\
Accuracy, \% recovery & 100.5 & 104.7 \\
Linearity, $r$ & 0.997 & 0.997 \\
LOD, $\mathrm{mg} / \mathrm{kg}$ & 0.0292 & 0.0014 \\
LOQ, $\mathrm{mg} / \mathrm{kg}$ & 0.0974 & 0.0046 \\
\hline
\end{tabular}


Table 3. Summary of statistical analysis for homogeneity of benzoic acid in mango juice.

\begin{tabular}{lccc}
\hline \multicolumn{1}{c}{ Criteria } & $\begin{array}{c}\text { Critical } \\
\text { Value }\end{array}$ & $\begin{array}{c}\text { Obtained } \\
\text { Result }\end{array}$ & Remarks \\
\hline $\begin{array}{l}\text { Cochran's Test, C } \\
\begin{array}{l}\text { Test for analytical } \\
\text { precision of the method }\end{array}\end{array}$ & 0.602 & 0.403 & Passed \\
$\begin{array}{l}\text { Test for sufficient } \\
\text { homogeneity }\end{array}$ & $<0.5$ & 0.125 & Passed \\
$\begin{array}{l}\text { Test for adequate } \\
\text { homogeneity }\end{array}$ & 14.25 & 0.437 & Passed \\
\hline
\end{tabular}

Table 4. Summary of statistical analysis for homogeneity of histamine in canned tuna.

\begin{tabular}{lccc}
\hline \multicolumn{1}{c}{ Criteria } & $\begin{array}{c}\text { Critical } \\
\text { Value }\end{array}$ & $\begin{array}{c}\text { Obtained } \\
\text { Result }\end{array}$ & Remarks \\
\hline $\begin{array}{l}\text { Cochran's Test, C } \\
\begin{array}{l}\text { Test for analytical } \\
\text { precision of the method }\end{array}\end{array}$ & 0.602 & 0.356 & Passed \\
$\begin{array}{l}\text { Test for sufficient } \\
\text { homogeneity }\end{array}$ & $<0.5$ & 0.473 & Passed \\
$\begin{array}{l}\text { Test for adequate } \\
\text { homogeneity }\end{array}$ & 0.602 & 33.395 & Passed \\
\hline
\end{tabular}

\subsection{Homogeneity and Stability}

The RMs prepared was found to be sufficiently homogeneous for benzoic acid and histamine studies. The results of the statistical evaluation for the homogeneity test of the benzoic acid and histamine RMs are shown in Table 3 and Table 4, respectively. Trend analyses for both RMs - used for shortterm stability evaluation - confirms that no significant instability of the materials were observed during transport at $4{ }^{\circ} \mathrm{C}, 30{ }^{\circ} \mathrm{C}$ and $40{ }^{\circ} \mathrm{C}$ for a period of three weeks. The long-term storage of the $\mathrm{RMs}$ at $4{ }^{\circ} \mathrm{C}$ was determined to be about 6 months for benzoic acid and 12 months for histamine. Figure 1 shows the plot of the long-term stability test for histamine in canned tuna.

\subsection{Reference value assignment}

The reference values assigned for benzoic acid in mango juice and histamine in canned tuna listed in Table 5 are the mean values of duplicate measurements from the three bottles from the batch, analysed by the LC-IDMS method. As part of quality assurance, the acceptable percentage recovery of the CRM HRM-1001A and control materials was achieved for benzoic acid and histamine, respectively.

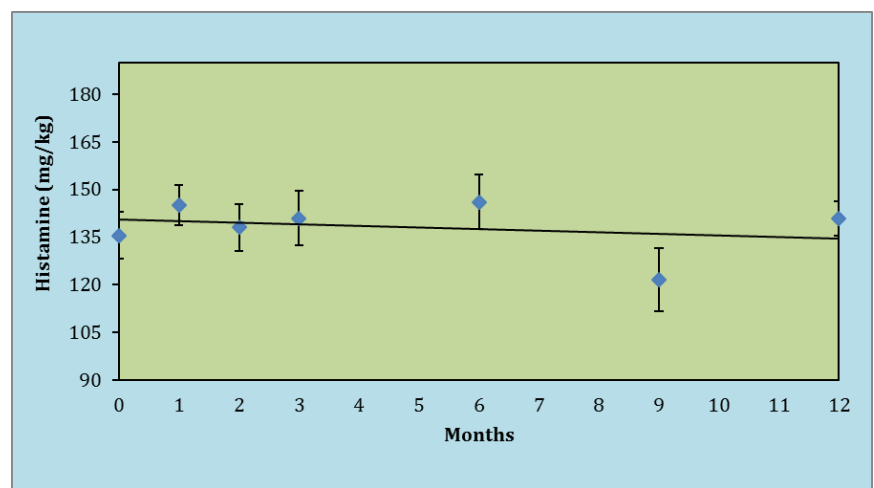

Figure 1. Long-term stability for histamine in canned tuna.
Table 5. Assigned value for the developed reference material.

\begin{tabular}{lccc}
\hline \multirow{2}{*}{ Reference Material } & \multicolumn{2}{c}{ Mass Fraction $(\mathrm{mg} / \mathrm{kg})$} & $\boldsymbol{n}$ \\
& Assigned Value & Uncertainty $(k=2)$ & \\
\hline $\begin{array}{l}\text { Benzoic acid in mango } \\
\text { juice }\end{array}$ & 774 & 42 & 6 \\
$\begin{array}{l}\text { Histamine in canned } \\
\text { tuna }\end{array}$ & 148 & 14 & 6 \\
\hline
\end{tabular}

For benzoic acid, MS was operated in electrospray ionisation in negative mode. Benzoic acid and its isotope analogue were detected by selectively monitoring the collision-induced dissociation channels of $[\mathrm{M}-\mathrm{H}]^{-} \rightarrow\left[\mathrm{M}^{-} \mathrm{CO}_{2} \mathrm{H}\right]^{-}$at $\mathrm{m} / \mathrm{z} 121.1 \rightarrow$ 77.2 and $\mathrm{m} / \mathrm{z} 126.1 \rightarrow 82.1$, respectively. For histamine, MS was operated in electrospray ionisation in positive mode. Histamine and its isotope analogue were detected by selectively monitoring the collision-induced dissociation channels of $[\mathrm{M}+\mathrm{H}]^{+} \rightarrow$ $\left[\mathrm{M}+\mathrm{H}-\mathrm{NH}_{3}\right]^{+} \rightarrow[\mathrm{M}+\mathrm{H}-\mathrm{CH} 2 \mathrm{CH} 2 \mathrm{NH} 2]^{+}$at $\mathrm{m} / \mathrm{z} 112.1 \rightarrow 95.1$ $\rightarrow 68.2$ and $\mathrm{m} / \mathrm{z} 116.1 \rightarrow 99.1 . \rightarrow 85.2$, respectively.

\subsection{Measurement of uncertainty}

The expanded uncertainties of the reference values of the $\mathrm{RMs}$ produced presented in Table 5 were estimated based on the ISO GUM approach [11]. The highest contribution to the expanded uncertainty of the reference values of RM benzoic acid (94.6\%) was obtained from the peak area ratio of sample-isotope blend, while homogeneity gave the highest contribution $(97.67 \%)$ for RM histamine in canned tuna. The remainder of the uncertainty sources included masses of samples, standards and solutions, and purity of the calibration standard.

\subsection{Participation in International supplementary comparison}

In 2014, in support of the reference value assignment capability, the Standards and Testing Division (STD) of ITDI the designated institute for metrology in chemistry in the country - participated in a supplementary comparison, APMP.QM-S8: Determination of mass fraction of benzoic acid, methyl paraben and n-butyl paraben in soy sauce [2]. This was coordinated by the Health Sciences Authority of Singapore under the auspices of the Organic Analysis Working Group (OAWG) of the Comité Consultatif pour la Quantité de Matière (CCQM). In this comparison, ITDI-STD demonstrated its competence for benzoic acid analysis in soy sauce.

\subsection{Conduct of Proficiency Test (PT) Scheme}

The RMs developed in this study were also used for accuracybased PT schemes, organised to assess the performance of local testing laboratories based on ISO/IEC 17043 [12]. The reference value obtained by a candidate higher order method was used as the assigned value for the PT scheme. The $\mathrm{z}$-score was based on the reference values of the RMs.

Government and private testing laboratories, research institutes and the academe participated in the PT schemes by using routine methods of analysis. In the PT round for benzoic acid in mango juice (MiCPT-14-01), most of the participants used HPLC, as shown in Figure 2. Nine (82\%) out of 11 laboratories obtained satisfactory performance, and two (18\%) obtained unsatisfactory results. The unsatisfactory results were observed to be due to computation error and overestimation of results due to matrix effects.

Furthermore, two different analytical techniques based on fluorometry and ELISA were used by twelve participants for the PT round for histamine (MiCPT-14-04). However, only $50 \%$ satisfactory performance was achieved. Results for this PT round 


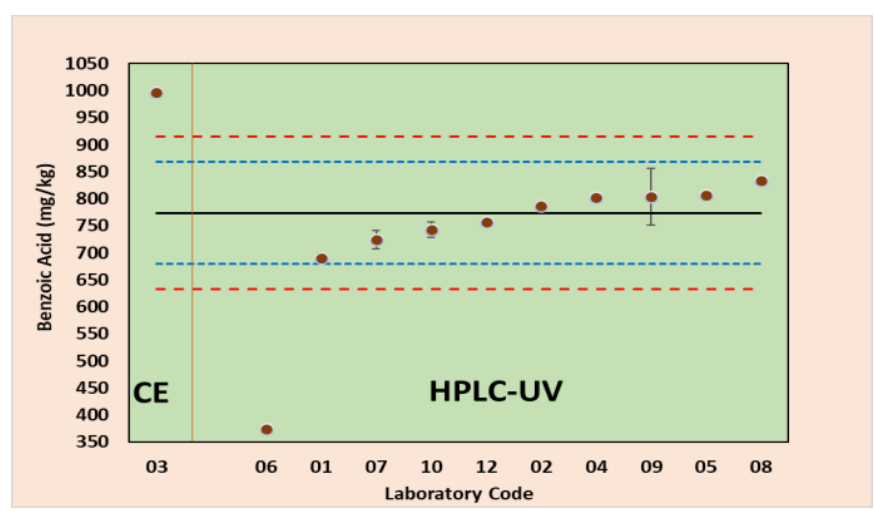

Figure 2. Participant results for MICPT-14-01: Benzoic acid in Mango Juice using capillary electrophoresis and high-performance liquid chromatography with UV detector.

are displayed in Figure 3, with reference to the sample preparation used prior to fluorometric detection. Under estimation of results were observed from four laboratories using the AOAC 977.13 method. The relative standard deviation of the participating laboratories with satisfactory performance is approximately $9 \%$. Different parameters for the extraction techniques and the need for validation of the method were identified problems in this PT scheme.

\section{CONCLUSIONS}

In this work, the capability to developed RMs for benzoic acid in mango juice and histamine in canned tuna, the provision of reference value to RMs using a candidate higher order method (LC-IDMS), and the conduct of PT schemes were demonstrated in the country through ITDI-STD. The prepared RMs were homogeneous and stable and were made available for the first time to local chemical testing laboratories. Through the PT exercises conducted using these RMs, an assessment for better performance by local laboratories for benzoic acid than for histamine analysis was observed. Likewise, this served as a means for our institute to disseminate the traceability of measurement to SI standards. The continuous development and use of RMs, PT schemes and method validation by the institute, along with local laboratories participating in PT schemes, are regarded as essential to further improving laboratory performance in the country and facilitating fair trade in food globally.

\section{ACKNOWLEDGEMENT}

Funding support for DOST and the Philippine Council for Industry, Energy and Emerging Technology for Research and Development (PCIEERD) is gratefully acknowledged. The authors are also thankful for the histamine standards provided by the National Institute for Metrology, China.

\section{REFERENCES}

[1] L. J. de Carvalhjo, $\quad$ E. C. Pires do Rego, $\quad$ B. C. Garrido, Quantification of benzoic acid in beverages: the evaluation and validation of direct measurement techniques using mass spectrometry, Anal. Methods 2016, 8, 2955-2960.

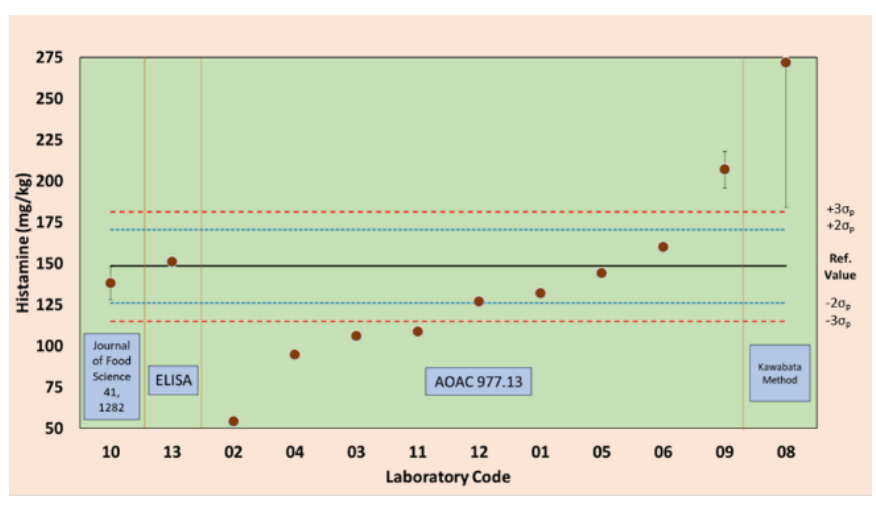

Figure 3. Participant results for MICPT-14-04: Histamine in canned tuna using fluorometer detection with different sample preparation.

[2] T. L. Teo, T. Lu， E. M. Gui， P. S. Cheow，P. Giannikopoulou, E. Kakoulides, E. Lampi，S. Choi，Y. Yip， P. Chan, S. Hui, W. Wollinger, L. Carvalho, B. Garrido, E. C. P. Rego, S. Ahn,

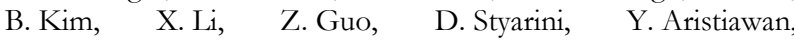
D. P. Ramadhaningtyas, N. Aryana, B. S. Ebarvia, A. Dacuaya, A. Tongson, K. Chr. Aganda, Th. J. Fortune, P. Tangtrirat, Th. Mungmeechai, A. C. Gören, S. Gündüz, H. Yilmaz, APMP.QM-S8: determination of mass fraction of benzoic acid, methyl paraben and n-butyl paraben in soy sauce, Metrologia 54(1A):08028-08028.

DOI: https://doi.org/10.1088/0026-1394/54/1A/08028

[3] I. Lee, S. Ahn, B. Kim, E. Hwang, Y. S. Kim, Analysis of benzoic in quasi-drug drink using isotope dilution liquid chromatography mass spectrometry, Bull. Korean Chem. Soc., 29 (2008), pp. 2125 2128

[4] Organic Chemistry Section - STD-ITDI (TM-OCS-202) unpublished in-house technical procedure for benzoic acid in fruit juice using HPLC with gravimetric sample preparation, 2015.

[5] F. B. Custodio, E. Tavares, M. B. A. Gloria, Extraction of bioactive amines from grated parmesan cheese using acid, alkaline and organic solvents, Journal of Food Composition and Analysis, 20 (2007), pp. 280-288.

[6] M. Thompson, S. R. Ellison, R. Wood, The International Harmonized Protocol for the Proficiency Testing of Analytical Chemistry Laboratories, Pure Appl. Chem., Vol. 78. No.1, pp. 145-196, 2006

DOI: https://doi.org/10.1351/pac200678010145

[7] ISO Guide 35:2006 Reference Materials - General and statistical principles for certification, (2006).

[8] H. Shin, B. Kim, J. Lee, E. Hwang, Development of an IsotopeDilution Liquid Chromatography/Mass Spectrometric Method for the Accurate Determination of Acetaminophen in Tablets, Bull. Korean Chem. Soc. 2010, Vol. 31, No. 12. DOI: https://doi.org/10.5012/bkcs.2010.31.12.3663

[9] N. Byrd, Quick, easy and reliable detection of histamine in food Using the Agilent 6490 Triple Quadrupole LC/MS with Jet Stream Technology, Agilent Application Note. 5991-1286EN, 2013.

[10] Official Methods of Analysis of AOAC International. AOAC International, Gaithersburg, MD, USA, 2012. 19th Ed., Vol. 2.

[11] Joint Committee Guides in Metrology, JCGM 100:2008, Evaluation of measurement data - Guide to expression of uncertainty in measurements (ISO GUM). $1^{\text {st }} \mathrm{Ed}$.

[12] ISO/IEC 17043: 2010 Conformity assessment - General requirements for proficiency testing, International Standards Organisation, Switzerland 